\title{
评述 复杂网络上的动力学专辑
}

\section{复杂网络上的爆炸式同步}

\author{
管曙光* \\ 华东师范大学物理与材料科学学院, 上海 200241 \\ *联系人, E-mail: sgguan@phy.ecnu.edu.cn
}

收稿日期: 2019-04-17; 接受日期: 2019-05-05; 网络出版日期: 2019-09-18

国家自然科学基金(编号: 11835003, 11875132)和上海市自然科学基金(编号: 18ZR1411800)资助项目

\begin{abstract}
摘要 耦合振子系统的同步是统计物理和复杂系统研究的重要领域之一. 过去几十年的研究表明, 该系统 中绝大多数同步化相变都是连续相变 (二级相变), 即序参量通过相变点后连续增长. 但是最近发现在某些耦 合振子系统中, 同步可以表现为爆炸式(一级相变). 其特征为序参量通过相变点后出现突然的跃变, 并且随 耦合强度变化, 向前和向后的相变过程不可逆, 存在磁滞区. 由于爆炸式同步化与连续相变同步化有着本质 上的不同, 所以这一发现开拓了新方向, 具有十分重要的意义. 本文综述了这一方向目前的重要成果, 主要 包括频率和度关联振子系统的爆炸式同步、频率权重Kuramoto模型中的爆炸式同步、拥护者和反对者耦 合网络中的爆炸式同步、多层网络上的爆炸式同步, 以及爆炸式同步的形成机制和实验验证等. 这些工作 不仅极大地加深了我们对于复杂系统同步化的认识, 促进同步理论的发展, 而且能够为将来在相关领域的 应用打下基础.
\end{abstract}

关键词 Kuramoto模型, 同步, 爆炸式同步, 相变, 复杂网络

PACS: 05.45.Xt, 68.18.Jk, 89.75.-k

\section{1 同步研究简介}

同步现象是指由两个以上的组元构成的系统 中, 通过组元之间的相互协作或竞争(物理上称为耦 合), 在宏观上形成步调一致的运动或节律. 同步现 象广泛存在于自然界和人类社会中. 例如大量苂火 虫的同步发光、蟋蟀和青蛙的同步鸣叫、音乐会观 众有节律的鼓掌、伦敦千禧桥与游客形成的共振、 心脏起搏细胞的同步振动、动植物的生理节律等都 是众所周知的同步现象. 更广义地讲, 激光、化学振 荡、流体对流斑图、股票市场的涨跌、传染病的爆
发以及社会公众與论的形成与传播等都存在着与同 步类似的协同行为.

据史料记载, 荷兰物理学家惠更斯于1665年最 早发现和研究了两个物理振子之间的同步现象. 他 发现两个悬挂于同一支架上的钟摆, 不管初始位 置如何, 最终它们总会达到步调一致, 即形成同步. 20世纪60年代以来, 随着非平衡统计领域自组织理 论和协同学的发展, 同步和协同行为在物理、化学、 生物、医学、工程技术以及社会经济等领域得到了 深入的研究和广泛的应用 ${ }^{[1,2]}$. 进入 20 世纪 90 年代, 混沌控制和同步的研究迅速发展: 完全混沌同步、

引用格式: 管曙光. 复杂网络上的爆炸式同步. 中国科学: 物理学 力学 天文学, 2020, 50: 010504 Guan S G. Explosive synchronization in complex networks (in Chinese). Sci Sin-Phys Mech Astron, 2020, 50: 010504, doi: 10.1360/SSPMA-2019-0135 
广义混沌同步、混沌相同步等现象被相继发现, 新 的概念、新的理论方法被相继提出, 新的应用得以 展示. 这些工作极大地丰富和发展了传统的同步化 研究 ${ }^{[3]}$. 进入 21 世纪以后, 随着国内外复杂网络研 究的迅猛发展, 复杂网络上的同步化研究又进入到 了一个新的层次 ${ }^{[4]}$. 迄今为止, 在具有不同类型拓 扑结构的各种复杂网络上, 如小世界网络、无标度 网络、加权网络、集团网络和梯度网络等, 同步化 现象得到了积极和深入的研究, 并且还在继续进行. 值得关注的是, 我国学者紧跟国际前沿, 在这一领域 取得了很多创新性的成果 ${ }^{[5]}$.

理论上, 耦合系统的同步化研究都是基于模型 开展的, 主要包括偏微分方程模型、常微分方程模 型和离散的原胞自动机模型等. 特别是对耦合振子 模型, 即耦合常微分方程模型, 研究得最多, 也最为 深入. 其中, 日本学者Kuramoto 在1975年提出了耦 合相振子模型——后来被命名为Kuramoto模型 ${ }^{[6,7]}$ :

$\dot{\theta}_{i}=\omega_{i}+\frac{\kappa}{N} \sum_{j=1}^{N} \sin \left(\theta_{j}-\theta_{i}\right), \quad i=1, \ldots, N$,

式中, $\theta_{i}$ 和 $\omega_{i}$ 分别为第 $i$ 个相振子的相位和本征(自 然)频率, $\kappa$ 为耦合强度. 通常情况下, 系统中所有振 子的本征频率满足某种分布 $g(\omega)$, 常见的频率分布 见表1.

为了描述系统宏观的协同行为, Kuramoto系统 可以定义一个序参量:

$r \mathrm{e}^{\mathrm{i} \psi}=\frac{1}{N} \sum_{j=1}^{N} \mathrm{e}^{\mathrm{i} \theta_{j}}$,
即所有振子复振幅的平均值(或平均矢量). $r$ 表 示序参量的大小 $(0 \leq r \leq 1), \psi$ 是序参量的相位. $r \approx 0$ 表示系统处于非协同态(也称无序态、混乱态 等), 而 $r \approx 1$ 表示系统处于协同态(也称有序态、同 步态等). 由于文献中也经常用 $R$ 表示序参量, 为了方 便起见, 本文不加区分, 两种表示通用. 根据序参量 的定义, Kuramoto模型可以写成平均场形式:

$\dot{\theta}=\omega+\kappa r \sin (\psi-\theta)$.

从平均场方程可以看出, 每一个振子只受平均场影 响, 相当于振子之间通过平均场进行耦合.

在Kuramoto模型中, 振子的动力学被高度简化, 只由相位来描述, 振幅动力学则完全被忽略, 如 图1(a)所示. 然而就是这样一个非常简化的模型却 能抓住同步化问题的本质, 可以描述许多实际耦合 系统的协同行为, 如萤火虫的发光同步、心肌细胞
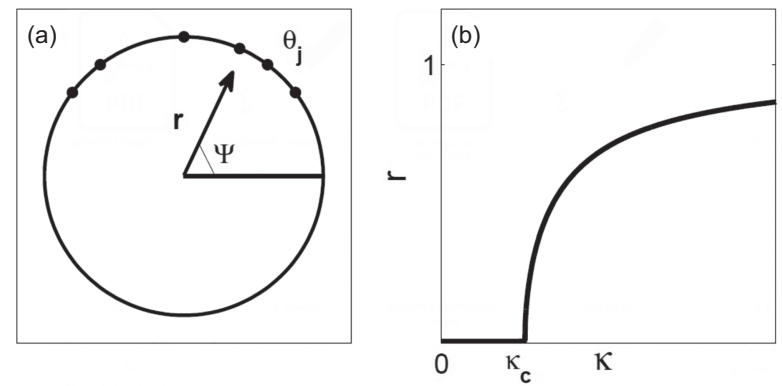

图 1 Kuramoto模型中相振子和序参量示意图(a)以及序参 量 $r$ 随耦合强度 $\kappa$ 的变化曲线(b), 显示系统发生连续同步相变

Figure 1 Shematic plot for oscillators and order parameter in $\mathrm{Ku}$ ramoto model (a) and order parameter $r$ vs coupling strength $\kappa$ (b), showing a continuous synchronization transition.

表 1 常见频率分布

Table 1 Typical frequency distributions

\begin{tabular}{ccc}
\hline 分布名称 & 表达式 $g(\omega)$ & $\Delta$ \\
\hline Lorentzian & $\frac{\Delta}{\pi\left(\omega^{2}+\Delta^{2}\right)}$ & $\Delta, \omega_{0} \neq 0$ \\
Asymmetric Lorentzian & $\frac{\Delta}{\pi\left[\left(\omega-\omega_{0}\right)^{2}+\Delta^{2}\right]}$ & $\Delta, \omega_{0}$ \\
Bimodal Lorentzian & $\frac{\Delta}{2 \pi}\left[\frac{1}{\left(\omega-\omega_{0}\right)^{2}+\Delta^{2}}+\frac{1}{\left(\omega+\omega_{0}\right)^{2}+\Delta^{2}}\right]$ & $\Delta$ \\
Gaussian & $g(\omega)=\frac{1}{\sqrt{2 \pi}} \exp \left(\frac{-\omega^{2}}{2 \Delta^{2}}\right)$ & $\Delta$ \\
Triangle & $\left(\Delta-\left|\omega-\omega_{0}\right|\right) / \Delta^{2},\left|\omega-\omega_{0}\right| \leq \Delta$ & $\Delta$ \\
Uniform & $1 /(2 \pi \Delta),|\omega| \leq \pi \Delta$ & $\Delta, \omega_{0}$ \\
Rayleigh & $\frac{\omega-\omega_{0}}{\Delta^{2}} \exp \left[-\frac{\left(\omega-\omega_{0}\right)^{2}}{2 \Delta^{2}}\right]$ & \\
\hline
\end{tabular}


的同步振动以及超导约瑟夫森节阵列的同步等. 正 因为如此, 该模型已经成为了同步研究领域最经 典和最成功的范例. 原始的Kuramoto模型中相振子 之间是全连的, 后来将相振子之间的耦合推广到 具有一定拓扑结构的情形, 即复杂网络, 统称为广 义Kuramoto模型.

对于更一般的耦合(全同)混沌振子的同步化问 题, Pecora和Carroll ${ }^{[8]}$ 给出了一个重要的理论框架, 也就是主稳定性分析方法(Master Stability Analysis). 该方法表明, 耦合振子系统能否达到同步化取决于 网络拓扑结构和振子动力学之间的相互作用. 概括 地讲, 由网络的拓扑结构定义的拉普拉斯矩阵的本 征值、振子之间耦合函数的形式以及振子本身的动 力学特性是影响同步化的关键因素 ${ }^{[8]}$.

过去几十年对耦合Kuramoto相振子以及耦合 混沌振子的研究表明: 在绝大多数情况下, 系统的 同步化, 即从非协同态向协同态的相变, 是二级相 变, 其中表征系统宏观协同运动的序参量通过相变 点后连续地增长 ${ }^{[9]}$. 以经典Kuramoto模型为例, 理 论表明, 在相变点附近序参量的增长满足幂律规 律, 幂律指数为 $1 / 2$, 如图 1 (b) 所示. 值得指出的是, 虽 然也有少数一级相变同步化的报道 ${ }^{[10-12]}$, 但不是 主流.

然而, 这个在过去一直为大家所认可的结论最 近受到了挑战. 2011 年, 西班牙Gómez-Gardeñes小 组 ${ }^{[13]}$ 在Physical Review Letters 上报道了他们的新发 现: 在一类广义Kuramoto模型中, 当网络的拓扑为 无标度网, 并且网络的节点度 (节点在网络中的邻居 数)与振子的本征频率严格相等时, 系统从非协同态 向协同态的同步化相变可以是一个突然的、爆炸 式的跃变, 称为爆炸式同步(Explosive Synchronization). 在爆炸式同步相变过程中, 序参量通过相变点 后有一跃变, 并且随着耦合强度变化, 其向前和向后 相变过程不可逆, 存在磁滞区, 这正是一级相变的 典型特征. 稍后, 在耦合Rössler振子模型中也发现 了同样的爆炸式同步化现象, 并且在星形网络上实 现了电路验证 ${ }^{[14]}$. 值得指出的是, 2011年Strogatz小 组 ${ }^{[15]}$ 研究了吸引和排斥耦合共存的Kuramoto模型, 即同时包含拥护者和反对者的耦合相振子系统. 他 们发现随着网络(全连网)中拥护者比例的增加, 系
统会表现出一级相变同步化.

Gómez-Gardeñes小组的开创性工作表明: 在一 定条件下, 耦合振子系统的同步化相变可以是一级 相变. 这一重要发现立刻引起了同步化研究领域中 学者们的极大关注. 按道理说, 一级相变和二级相 变都是统计物理中早已为人们所耳熟能详的基本 概念, 再发现一种一级相变现象也不应该是非常令 人惊奇的事情. 然而, 爆炸式同步为什么迅速成为前 沿的热点呢? 这里有以下几个原因. 第一, 自1998年 提出小世界网络和无标度网络以来, 复杂网络的研 究成为了非线性物理和复杂系统研究的主流 ${ }^{[5,16]}$. 当然网络上的同步研究也取得了很多进展, 包括形 成了主稳定性分析(Master Stability Analysis)等理论 成果, 然而复杂网络上观察到的同步绝大多数都是 连续相变. 第二, 传统上, 渗流是一种连续的几何相 变. 然而2009年发现, 在Achlioptas过程中(Achlioptas Process) ${ }^{[17]}$, 渗流可以是一级相变, 即爆炸式渗流. 2011年后, Gómez-Gardeñes小组的工作与此极其类 似, 也是在一个之前基本都是连续相变的领域发现 了一级相变, 并且爆炸式同步的名称应该也受到了 爆炸式渗流的影响. 第三, 一级相变同步化具有很强 的现实意义. 在我们生活的物理世界中, 复杂系统的 同步化或协同行为在很多情况下就表现为随系统某 种控制参量变化而突然出现跃变的现象. 例如, 现代 社会严重依赖的电力网络在某些情况下可能因小事 故发生级联效应而崩溃, 从而导致发生大规模停电 事故, 给国民经济和人民生活造成重大损失和影响; 互联网有时会进入大规模的拥堵状态, 严重影响信 息的传递; 某些物种在环境或资源变化下可能从稳 定态通过一级相变跳到灭绝态. 在名著《协同学》 中, Haken ${ }^{[18]}$ 指出, 社会以及经济系统中存在着与物 理系统类似的协同行为. 有时这类行为可以是系统 在两个不同性质的态之间的迅速转变, 例如股票和 证券市场的剧烈波动、社会舆论乃至国家政治制度 的突然转变等. 长久以来, 人们缺乏一个描述此类 系统中宏观态随控制参量跃变的理论模型, 而爆炸 式同步化的发现则正好填补了这方面的空白. 人们 希望基于此开展相关的研究, 加深我们对于此类一 级相变动力学的理解, 从而指导实际问题中的应用. 基于以上的原因, 爆炸式同步化迅速成为复杂系统 
研究前沿的一个热点, 在短短的几年时间内已经取 得了诸多实质性进展, 本文将对这一领域的重要成 果进行综述、梳理、总结和展望. 希望能够抛砖引 玉, 为同行学者和研究生们提供参考.

\section{2 节点度和振子频率相关的网络爆炸式 同步}

\section{1 无标度网络}

2011年, Gómez-Gardeñes等人 ${ }^{[13]}$ 在Physical Review Letters发表文章“Explosive synchronization transitions in scale-free networks”, 这是爆炸式同步这 一术语首次出现. 稍早些时候(自2009年起), 另外 一种复杂网络上的几何(拓扑)相变——爆炸式渗 流(Explosive Percolation)正在被热烈争论. 限于篇 幅, 本文仅综述爆炸式同步方面的重要进展, 对于其 他的爆炸式相变, 可参考综述文献[16].

文献[13]研究的是复杂网络上的经典Kuramoto 模型, 其动力学方程为

$\dot{\theta}_{i}=\omega_{i}+\frac{\lambda}{N} \sum_{j=1}^{N} A_{i j} \sin \left(\theta_{j}-\theta_{i}\right), \quad i=1,2, \ldots, N$,

式中, $\theta_{i}$ 和 $\omega_{i}$ 分别为第 $i$ 个相振子的相位和本征频率, $\lambda$ 为耦合强度; $A_{i j}$ 为振子连接矩阵的矩阵元. 如果振 子 $i$ 和 $j$ 之间有连接, 则 $A_{i j}=1$, 否则为 0 . 在此基础上, 文献[13]作出了两条假设: (1) 振子连接形成无标度 网络; (2) 如果第 $i$ 个振子所处的网络节点度为 $k_{i}$, 则 该振子的本征频率 $\omega_{i}=k_{i}$. 这里实际上就是引入了 网络结构 $\left(k_{i}\right)$ 和振子动力学 $\left(\omega_{i}\right)$ 之间的关联. 如图 2 所 示, 结果表明, 在满足这两个假设的条件下, 系统 的序参量随耦合强度的变化有一个突然的跃变, 并 且向前相变点和向后相变点不一致, 向后相变点小 于向前相变点, 即向前和向后相变不可逆, 形成一 个磁滞区(图2(d)). 显然, 这里的同步相变不同于经 典Kuramoto模型的连续相变(二阶相变), 而是典型 的不连续相变, 即一阶相变. 文献[13]形象地将之命 名为“爆炸式同步”, 这个名称一直沿用至今.

文献[13]从理论上给出了爆炸式同步的一个近 似解释. 将无标度网络简化为星形网络(一个中心节
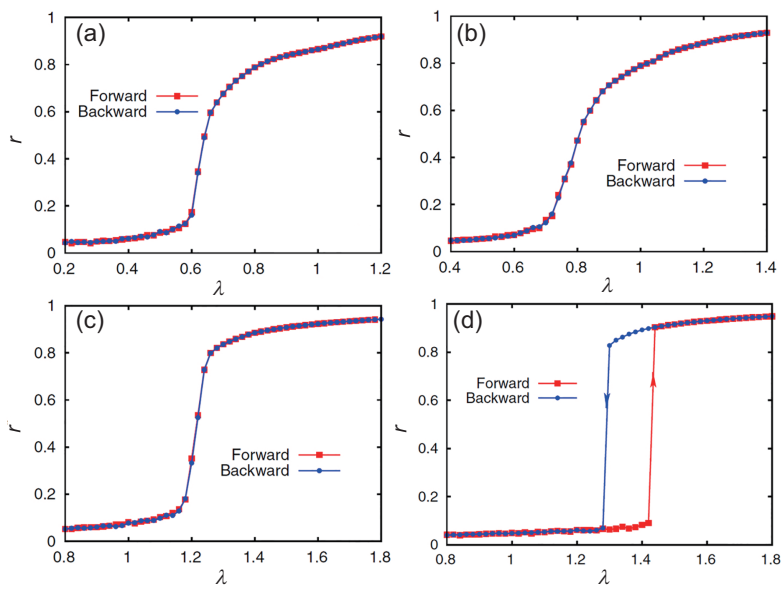

图 2 (网络版彩图)爆炸式同步化. 图中显示了在4种网络 上序参量 $r$ 随耦合强度 $\lambda$ 的变化曲线. 网络的控制参数为 $\alpha$ $(\alpha \in[0,1]), N=10^{3},\langle k>=6$. (a) $\alpha=1$, 即ER网络; (b) $\alpha=0.6 ;$ (c) $\alpha=0.2 ;$ (d) $\alpha=0$, 即BA网络. 图中用绝热方式研 究了向前和向后的相变. 改编自参考文献[13], 版权归美国物 理学会所有

Figure 2 (Color online) Explosive synchronization. Order parameters $r$ vs coupling strength $\lambda$ have been plotted for four network topologies, characterized by parameter $\alpha(\alpha \in[0,1]) . N=10^{3},<k>=6$. (a) $\alpha=1$, i.e., ER network; (b) $\alpha=0.6$; (c) $\alpha=0.2$; (d) $\alpha=0$, i.e., BA network. Both forward and backward transitions have been investigated in an adiabatic way. Adapted with permission from ref. [13]. Copyrighted by the American Physical Society.

点连接 $K$ 个周边节点), 可以解出向后相变点为 $\lambda_{\mathrm{b}}=$ $(K-1) /(K+1)$, 该点处序参量为 $r_{c}=K /(K+1) \sim 1$.

\section{2 星形网络}

Zou等人 ${ }^{[19]}$ 进一步深入研究了星形网络上的爆 炸式同步, 并且同样地采用了度和频率的关联假设, 即假设星形网络有 $K$ 个周边节点, 周边节点上的振 子本征频率为 $\omega_{j}=\omega, 1 \leq j \leq K$; 中心节点的度为 $K$, 本征频率为 $\omega_{K+1}=\omega K$. 系统的动力学方程为

$\dot{\phi}_{K+1}=K \omega+\kappa \sum_{j=1}^{K} \sin \left(\phi_{j}-\phi_{K+1}\right)$,

$\dot{\phi}_{j}=\omega+\kappa \sin \left(\phi_{K+1}-\phi_{j}\right), \quad 1 \leq j \leq K$.

此系统的同步流形为 $\phi_{1}=\phi_{2}=\cdots=\phi_{K}, \phi_{K+1}-\phi_{1}=a$, 这 里 $a$ 是一个常数. 通过对同步流形存在的条件进行 分析, 可以得到向后相变点为 $\kappa_{\mathrm{b}}=(K-1) \omega /(K+1)$. 此外, 文献[19]通过数值计算表明当耦合强度大于 
向前相变点时, 即 $K>\kappa_{\mathrm{f}}$, 同步流形是全局吸引的. 然 后应用文献[20]的方法, 将中心振子和周边振子之 间的同步看成是完全同步的微扰, 可以解出向前相 变点近似为

$\kappa_{\mathrm{f}} \sim \frac{1}{B} \frac{K-1}{\sqrt{K}} \omega$,

式中, $B$ 是一个与初始条件有关的常数.

关于星形网络上频率和度关联的振子系统向前 相变点的求解, 除了文献[19]的方法之外, 也可以利 用动力学系统降维方法以及Ott-Antonsen方法来进 行求解, 具体可参考文献[21-23].

\section{3 自组织相关}

之前形成爆炸式同步的模型都采用了节点度和 本征频率相关的假设, 文献[24]的研究表明: 这一关 联可以通过系统的自组织形成, 从而放宽了这一约 束. 在文献[24]中, 复Ginzburg-Landau振子通过耗散 耦合, 其动力学方程为

$\dot{A_{i}}=A_{i}-(1+\mathrm{i} \alpha)\left|A_{i}\right|^{2} A_{i}+\kappa(1+\mathrm{i} \beta) \sum_{j=1}^{N} C_{i j}\left(A_{j}-A_{i}\right)$,

式中, $i=1,2, \ldots, N, A_{i}$ 为振子的复振幅, $C_{i j}$ 为振子 连接矩阵元, $\kappa$ 为耦合强度. $\alpha$ 和 $\beta$ 是无耦合时振子动 力学参数, 其中 $\alpha$ 为振子的本征频率.

文献[24]仅假设了振子连接形成无标度网络, 而振子为全同, 即本征频率都一样. 当耦合强度不 为 0 时, 文献[24]首先通过傅里叶变换计算每一个振 子时间序列的功率谱, 发现每一个振子有一个主 频 $\omega_{\mathrm{p}}$. 非常有趣的是, 随着耦合强度从 0 开始增加, 振子之间的相互作用加强, 原来相同的频率变得不 均匀, 形成一个分布, 并且与振子所在节点的度正相 关(如图3所示)! 这一结果表明, 之前研究爆炸式同 步时人为引入的节点度和振子频率的正相关这一条 假设可以放宽, 某些条件下系统可以通过相互作用 自组织来形成这一条件.

\section{3 频率权重Kuramoto模型中的爆炸式同步}

\section{1 频率权重Kuramoto模型}

早期的爆炸式同步都是在度和频率相关联的振

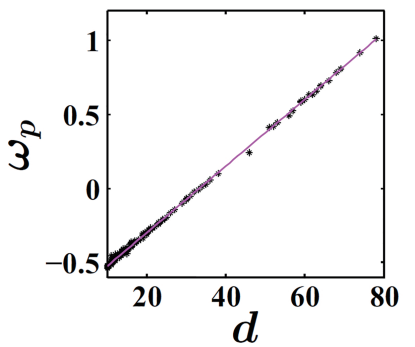

图 3 (网络版彩图) 振子的主频 $\omega_{\mathrm{p}}$ 与节点度 $d$ 通过自组织形 成正相关. 方程(8)的参数为 $\alpha=0.75, \beta=-1.7, N=200$, 网络平均度 $\langle d\rangle=20$. 在此参数下, 系统的向前相变点 为 $\kappa_{\mathrm{f}}=0.01$, 图中的参数 $(\kappa=0.0092)$ 非常靠近相变点. 点为 数值计算结果, 实线为理论结果, 二者符合得很好. 改编自参 考文献[24], 版权归美国物理学会所有

Figure 3 (Color online) Correlation is self-organized between the principle frequency $\omega_{\mathrm{p}}$ and the degree. Eq. (8) is integrated with parameters $\alpha=0.75, \beta=-1.7, N=200$, and the average degree $\langle d\rangle=20$. In this case, the forward transition point is $\kappa_{\mathrm{f}}=0.01$ and the parameter $(\kappa=0.0092)$ for the figure is very close to this point. The dots are numerical result and the solid line is the theoretical prediction. They are well consistent with each other. Adapted with permission from ref. [24]. Copyrighted by the American Physical Society.

子系统中发现的, 而且要求网络具有高度异质性, 即 无标度网络或星形网络. 这种对网络拓扑性质的要 求极大地限制了爆炸式同步的理论研究. 能否给出 一个不依赖于特殊网络的爆炸式同步理论模型? 为 此, 文献[25]提出了频率权重耦合的Kuramoto模型. 该模型的动力学方程为

$\dot{\theta}_{i}=\omega_{i}+\frac{\kappa\left|\omega_{i}\right|}{\sum_{j=1}^{N} A_{i j}} \sum_{j=1}^{N} A_{i j} \sin \left(\theta_{j}-\theta_{i}\right), \quad i=1, \ldots, N$.

这里的变量定义与方程(4)一致. 与经典Kuramoto模 型相比, 最重要的就是在耦合强度上引进了频率权 重因子. 这一因子使得系统的耦合不再均匀, 而是 具有异质性. 正是这一本质性的改变, 导致了模型 出现爆炸式同步行为. 如图4所示, 系统可以在不同 的网络拓扑下形成爆炸式同步, 从而不再局限于无 标度网络.

事实上, 频率权重Kuramoto模型与方程(4)在平 均场近似下是一致的. 从方程(4)可以看出, 在平均 场近似下, 每一个振子从邻居接收到的相互作用 正比于该振子的度, 而在节点度和振子频率相等的 假设下, 振子的有效耦合就相当于正比于其频率. 因 


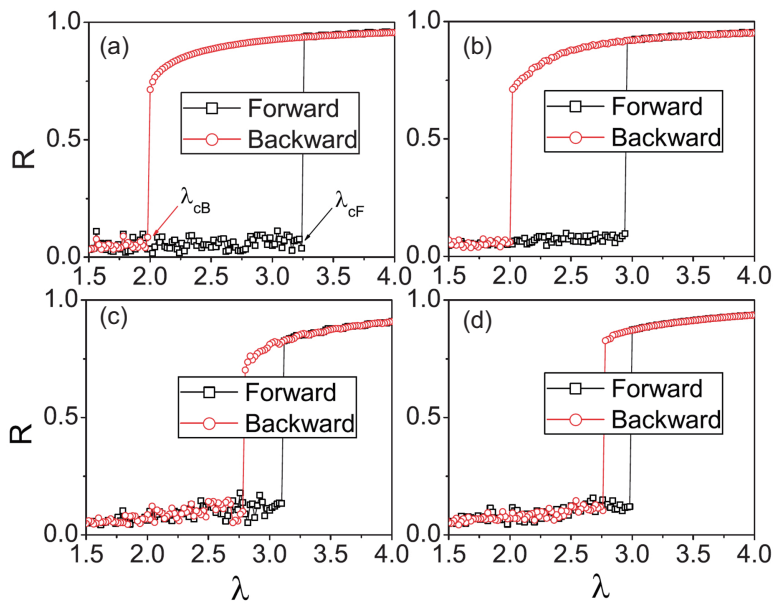

图 4 (网络版彩图)不同网络下频率权重Kuramoto模型中的 爆炸式同步. $N=500,<k>=6$. (a) 全连网, 本征频率为洛伦 兹分布; (b) 全连网, 本征频率为高斯分布; (c) ER 网, 本征频 率为洛伦兹分布; (d) UCM 网, 本征频率为洛伦兹分布. 改编 自参考文献[25],版权归美国物理学会所有

Figure 4 (Color online) Explosive synchronization in frequencyweighted Kuramoto model with various network topologies. $N=500$, $<k>=6$. (a) Fully connected network and Lorentzian frequency distribution; (b) fully connected network and Gaussian frequency distribution; (c) ER network and Lorentzian frequency distribution; (d) UCM network and Lorentzian frequency distribution. Adapted with permission from ref. [25]. Copyrighted by the American Physical Society.

此, 两种模型中都能出现爆炸式同步是意料之中, 但 是频率权重Kuramoto模型能够在各种网络拓扑下 产生爆炸式同步, 特别是在全连网上, 这是这个模 型的优点之一, 也是后来其得到广泛而深入研究的 原因.

\section{2 频率权重Kuramoto模型的相变点求解}

文献[25]的研究表明: 频率权重Kuramoto模型 能形成爆炸式同步, 并且不依赖于特定的网络结构. 特别是在全连网上也能出现爆炸式同步, 这给从理 论上精确求解相变点带来了便利. 因为无标度网和 星形网是节点度分布具有高度异质性的网络, 平均 场理论一般不太容易应用. 即使想办法进行理论处 理, 也只能采用大量近似, 文献[13,19]等就是如此.

$\mathrm{Hu}$ 等人 ${ }^{[26]}$ 系统地研究了全连网上的频率权 重Kuramoto模型, 给出了多种常见频率分布下的向 前和向后的相变点的精确解. 全连网上的频率权
重Kuramoto模型可表示为

$\dot{\theta}_{i}=\omega_{i}+\frac{\kappa\left|\omega_{i}\right|}{N} \sum_{j=1}^{N} \sin \left(\theta_{j}-\theta_{i}\right), \quad i=1, \ldots, N$.

为了求解爆炸式同步的向前相变点, 文献[26] 采用了Strogatz ${ }^{[7]}$ 提出的用几率密度描述振子同步 化的方法. 其基本思想如下: 在热力学极限下, 即 振子数目 $N \rightarrow \infty$ 的条件下, 可以引入一个几率密度 函数 $\rho(\theta, \omega, t)$. 其物理意义是: $\rho(\theta, \omega, t) \mathrm{d} \theta$ 表示频率 为 $\omega$ 、时刻为 $t$ 、相位在 $\theta$ 和 $\theta+\mathrm{d} \theta$ 之间的振子的比例. 该几率密度函数对所有 $\omega$ 和所有时刻 $t$ 满足归一化 条件:

$\int_{0}^{2 \pi} \rho(\theta, \omega, t) \mathrm{d} \theta=1$,

以及连续性方程

$\frac{\partial \rho}{\partial t}+\frac{\partial(\rho \dot{\theta})}{\partial \theta}=0$.

平均场下系统式(10)的动力学方程可表示为

$\dot{\theta}=\omega+\kappa|\omega| r \sin (\phi-\theta)$,

式中, $r$ 和 $\phi$ 为序参量. 按照文献[7]的处理方法, 对系 统的非协同态进行线性稳定性分析, 可以得到描述 非协同态失稳的特征方程:

$1=\frac{\kappa}{2} \int_{-\infty}^{+\infty} \frac{|\omega|}{\lambda+\mathrm{i} \omega} g(\omega) \mathrm{d} \omega$.

这一方程将刻画非协同态失稳的本征值 $\lambda$ 与耦合强 度 $\kappa$ 联系了起来. 应用非协同态失稳的条件并且注意 到这里本征值 $\lambda$ 并非像经典Kuramoto模型那样一定 为实数, 对于典型的单峰洛伦兹分布(见表1), 可以 求得向前相变点的精确解为 $\kappa_{\mathrm{f}}=4$.

对于向后相变点, 可以应用自洽方法求解. 根 据平均场方程(13), 假设系统一开始处于协同态并 且满足 $r k \geq 1$, 可以得到系统的定态解为

$\theta^{*}= \begin{cases}\theta_{\mathrm{p}}=\arcsin \frac{1}{\kappa r}, & \omega \geq 0, \\ \theta_{\mathrm{n}}=\arcsin \frac{-1}{\kappa r}, & \omega<0 .\end{cases}$ 
物理上这个解对应系统的协同态, 包括两个同步集 团, 其相位分别为 $\theta_{\mathrm{p}}$ 和 $\theta_{\mathrm{n}}$. 进一步可以求出协同态对 应的序参量为

$r_{1}(\kappa)=\frac{\sqrt{2}}{2} \sqrt{1+\sqrt{1-4 / \kappa^{2}}}, \quad \kappa \geq 2$,

$r_{2}(\kappa)=\frac{\sqrt{2}}{2} \sqrt{1-\sqrt{1-4 / \kappa^{2}}}, \quad \kappa \geq 2$.

对协同态的线性稳定性分析表明, $r_{1}$ 这支解(包含两 团振子)是稳定的, 而 $r_{2}$ (包含两团振子)是不稳定的. 从 $r_{1}$ 和 $r_{2}$ 的解析表达式可以看出, 这两支解存在的 范围是 $K \leq 2$, 因此向后相变点 $\kappa_{\mathrm{b}}=2$.

以上分析结果如图5所示. 图5(a)显示了洛伦兹 频率分布下(见表1), 系统向前和向后相变形成的 磁滞区, 向前相变点为 $\kappa_{\mathrm{f}}=4$, 向后相变点为 $\kappa_{\mathrm{b}}=2$, 数值结果与理论预测一致. 图5(b)显示了系统的 向后相变情况. 当系统耦合强度足够大 $(\kappa \sim \infty)$, 稳定解和不稳定解分别靠近实轴 $\left[r_{1}(\infty) \approx 1\right]$ 和虚 轴 $\left[r_{2}(\infty) \approx 0\right]$. 随着耦合强度的减小, 两个解相互靠 近, $r_{1}$ 减小而 $r_{2}$ 增大, 如图5(a)所示. 当耦合强度到达 相变点 $\kappa_{\mathrm{b}}=2$ 时, 两个解在 $\theta= \pm \pi / 4$ 处相撞而消失,
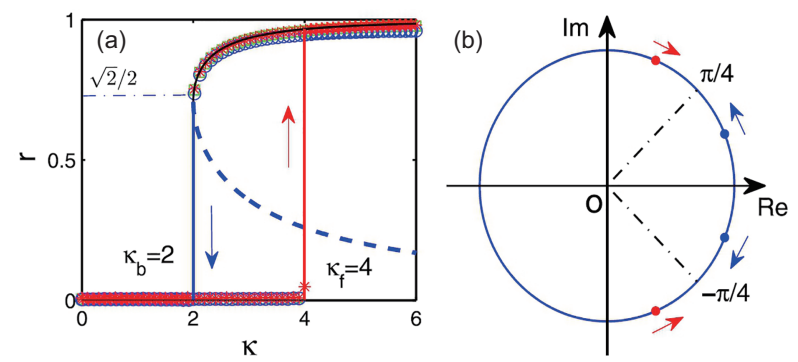

图 5 (网络版彩图) (a) 洛伦兹频率分布下方程(10)的爆炸式 同步. $N=20000, \Delta=1.0,2.0,3.0$. 系统有两支同步解: 蓝 色实线为稳定解 $r_{1}$, 蓝色虚线为不稳定解 $r_{2}$. (b) 向后相变的 分岔示意图. 当耦合强度足够大时, 稳定解(蓝点)和不稳定 解(红点)分别处于靠近实轴和虚轴的位置, 随着耦合强度的 减小, 它们相互靠近并最终在 $\theta= \pm \pi / 4$ 处碰撞而消失, 对应 的向后相变点 $\kappa_{\mathrm{b}}=2$

Figure 5 (Color online) (a) The explosive synchronization of eq. (10) with Lorentzian frequency distribution. $N=20000$ and $\Delta=1.0,2.0,3.0$, respectively. There are two coherent solutions: stable $r_{1}$ (blue line) and unstable $r_{2}$ (dashed line). (b) The schematic plot for the backward bifurcation. When the coupling strength is large enough, the stable (blue dot) and unstable coherent (red dot) solutions are located near the real and imaginary axes, respectively. With the decrease of the coupling strength, they move oppositely along the unit circle. Eventually, they collide and disappear at $\theta= \pm \pi / 4$, corresponding to $\kappa_{\mathrm{b}}=2$.
因此向后相变整体是一个鞍结分岔的图像.

以上分析同时揭示了频率权重Kuramoto模型 中出现爆炸式同步的机制. 对比经典Kuramoto模 型和频率权重Kuramoto模型的平均场方程, 即方 程(3)和(13), 可以发现前者的耦合强度是均匀的, 当 振子频率分布不均匀时, 形成同步集团是一个渐 进的过程. 频率小的振子先被平均场驯服, 而频 率大的振子需要较大的平均场才能拉进同步集团. 这样, 经典Kuramoto模型的同步相变就表现为序参 量随耦合强度逐渐增加的连续相变. 相反, 频率权 重Kuramoto模型平均场方程(13)中, 耦合强度是异 质性的, 且正比于振子的本征频率, 这样频率大的 振子其受到的有效耦合强度也成正比地变大. 当耦 合强度足够大时, 不管振子的本征频率大小如何, 它们只能一起形成同步. 换言之, 振子要么都不同 步(非协同态), 要么都一齐同步(协同态), 不存在像 经典Kuramoto模型那样的部分同步态.

文献[26]系统地研究了其他频率分布的情形, 如三角分布、高斯分布等(见表1), 理论上向前相变 点都可以通过方程(14)求解出来. 另外, 研究发现不 管振子为何种频率分布, 系统的向后相变点理论上 都是 $K_{\mathrm{b}}=2$. 这一结果并不奇怪, 其实正是频率权重 模型的特点: 因为振子的有效耦合强度与频率成正 比, 有效耦合强度与频率分布差异性形成的效果抵 消, 因此是何种频率分布就不起作用了.

值得指出的是，文献[27]进一步预测了方 程(10)所有的协同态. 除了文献[26]给出的两集团 振子协同态之外, 还有行波态和驻波态. 此外, 文 献[27]还严格证明了频率权重Kuramoto模型在同步 相变点之前, 非协同态是中性稳定, 而不是线性稳定 的, 并且在非协同态是中性稳定区, 序参量存在指 数衰减的行为, 即著名的朗道阻尼现象 ${ }^{[28]}$. 此外, 文 献[29]将频率权重因子推广到关于频率的权重函数; 文献[30]研究了社区网络上的频率权重模型. 最近, 文献[31]研究了时滞下频率权重Kuramoto模型中的 爆炸式同步.

\section{3 双峰分布下的频率权重Kuramoto模型}

文献[32]详细研究了双峰洛伦兹分布下的频率 
权重模型(见表1). 在在文献[32]的附加材料中, 给出 了向前相变点的详细推导过程, 其解析解为

$\kappa_{\mathrm{f}}=\frac{4}{1+\left(\omega_{0} / \Delta\right)^{2}}$.

可见, 向前相变点只与约化参数 $\omega_{0} / \Delta$ 有关. 至于向 后相变点, 则依然是 $\kappa_{\mathrm{b}}=2$, 与具体频率分布无关. 图6给出了数值模拟结果和理论预测的对比, 二者完 全符合. 最近, 文献[33]研究了紧致双峰分布下的爆 炸式同步。

\section{4 均匀分布下的频率权重Kuramoto模型}

文献[27,34]研究了均匀分布下(见表1)频率权 重Kuramoto的同步相变. 图7给出了 $\Delta=0.5$ 时系统 的相变图. 由图可见, 均匀分布下系统的相变很有 特点, 与洛伦兹分布(图5)图像不同. 系统的向后相 变点依然为 $\kappa_{\mathrm{b}}=2$, 但向前相变点 $\kappa_{\mathrm{f}}<\kappa_{\mathrm{b}}=2$. 这样 随着耦合强度的增加, 系统首先经历一个跳变, 从 非协同态分岔到一个高阶协同态(即下一节将要介 绍的Bellerophon态), 然后再经过一次跳变进入协同 态. 向后相变也有两次, 同样地均为一阶相变.

\section{5 非对称分布下的频率权重Kuramoto模型}

经典Kuramoto模型具有旋转对称性, 即在变换

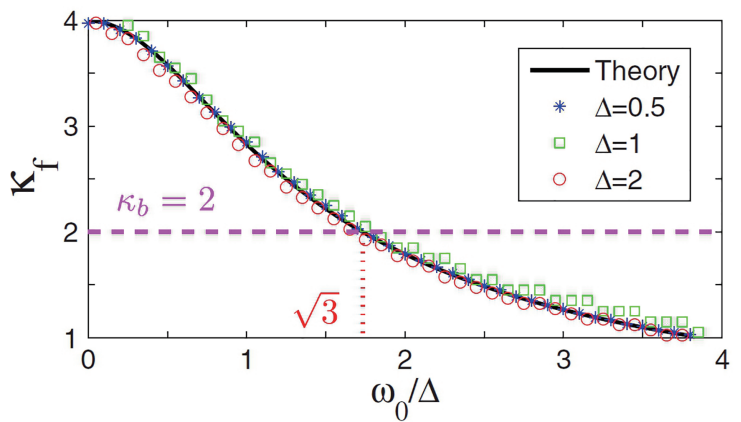

图 6 (网络版彩图)双峰洛伦兹分布下频率权重Kuramoto模 型的向前相变点 $\kappa_{\mathrm{f}}$ 随 $\omega_{0} / \Delta$ 的变化曲线. 黑线为解析解, 紫色 的虚线对应向后相变点. 理论预测与数值模拟结果符合得 很好

Figure 6 (Color online) Critical point for the forward transition in frequency-weighted Kuramoto model with bimodal Lorentzian distribution. $\kappa_{\mathrm{f}}$ vs $\omega_{0} / \Delta$. The black curve corresponds to the analytic solution. The purple dashed line marks the backward transition point. The theoretical prediction and the numerical results coincide perfectly.
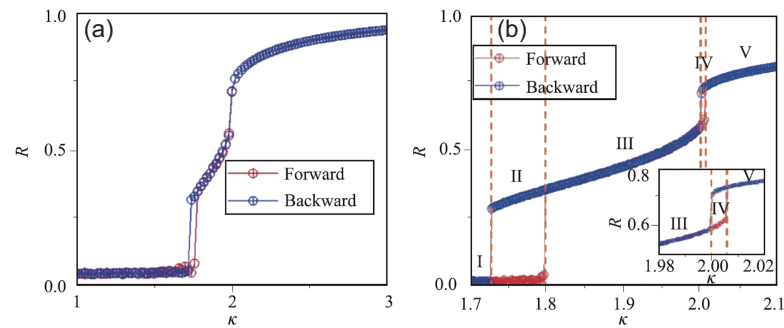

图 7 (网络版彩图)均匀分布下频率权重Kuramoto模型的同 步相变, $N=10000, \Delta=0.5$. (a) 序参量随耦合强度变化曲线. (b)为(a)图的放大图, 清晰显示出两次一级相变的磁滞区

Figure 7 (Color online) Synchronization transition in frequencyweighted Kuramoto model with uniform distribution. (a) Order parameter $R$ vs coupling strength $\kappa$. (b) Enlargement of (a) that clearly shows two hysteresis loops of first-order transition.

$\theta_{j}=\theta_{j}^{\prime}+\Omega t, \omega_{j}=\omega_{j}^{\prime}+\Omega$ 下, 系统的动力学方程 保持不变. 这样, 只要是本身对称的频率分布, 我 们总可以找到一个合适的旋转坐标系, 使得频率 分布关于零点对称. 因此, 对于经典Kuramoto模型, 一般只需要研究对称分布即可. 但是, 因为频率权 重Kuramoto模型在耦合强度上引入了频率因子, 所 以上述的旋转对称性不复存在. 这样, 对于频率权 重Kuramoto模型而言, 非对称频率分布下系统的同 步相变是一个值得研究的问题, 这正是文献[35]完 成的工作. 在文献[35]中, 我们详细研究了4种非对 称频率分布下频率权重Kuramoto模型的相变, 包括 非对称的洛伦兹分布、高斯分布、三角分布，以 及本身为固有非对称 (不能通过平移坐标轴变成对 称)的瑞利分布(见表1). 研究结果表明, 非对称频率 分布下, 频率权重Kuramoto模型在合适的参数下可 以表现出爆炸式同步, 当然也可以形成连续同步相 变. 由于非对称分布的特点, 同步相变后系统将普遍 形成行波态.

\section{6 爆炸式同步的微观机制}

渗流是一类几何相变, 关注的是在网络中如何 形成一个大的连通集团, 其规模可以和整个网络 的大小相比拟. 之前的渗流相变多为连续相变, 直 到2009年, Achlioptas等人 ${ }^{[17]}$ 在网络增加连边的过 程中引入了竞争机制, 即所谓的Achlioptas过程, 这 才发现了爆炸式渗流, 并迅速引起了广泛的关注. 
研究表明, 由于Achlioptas过程从微观上阻碍了网络 中形成较大的集团, 因此导致爆炸式渗流的产生. 受到这一研究的启发, 文献[36]比较了爆炸式渗流 和频率权重Kuramoto模型中爆炸式同步这两种相 变过程, 认为后者可以看成是状态空间的渗流, 并且 二者的微观形成机制相似.

具体地讲, 文献[36]定义了描述两个振子同步 的局域序参量:

$R_{i j}=\left|\lim _{T \rightarrow \infty} \int_{t}^{t+T} \mathrm{e}^{\mathrm{i}\left[\theta_{i}(t)-\theta_{j}(t)\right]}\right|$.

这里 $T$ 是一个足够长的时间窗口. 然后通过分析, 给 出了两个振子之间形成锁相的必要条件为

$\frac{\left|\omega_{i}-\omega_{j}\right|}{\left|\omega_{i}\right|+\left|\omega_{j}\right|} \leq \lambda R$

式中, $\lambda$ 为耦合强度. 此式即为一对振子间形成同步 的禁止律(Suppressive Rule). 当耦合强度小于临界 点时, 序参量 $R$ 非常小, 因此只有那些本征频率差满 足禁止律的振子才能形成同步集团. 因此这一条件 有利于形成小规模的同步集团, 有利于同步集团吸 引游离振子, 但是不利于小同步集团融合成更大的 集团. 当所有的游离振子都加入了同步集团, 继续 增加耦合强度就会导致之前形成的众多的、规模比 较小的同步集团突然合并, 形成巨大的同步集团, 即 发生爆炸式同步. 从微观上看, 爆炸式同步和爆炸式 渗流的形成机制非常相似. 非常引入注目的是, 爆 炸式同步源于禁止律的存在这一理论结果已被文 献[37]中人脑的意识与无意识实验所验证.

禁止律的发现同时为控制爆炸式同步提供了思 路: 当物理条件或控制手段有利于禁止律时, 系统 将形成爆炸式同步; 反之, 如果破坏禁止律的形成条 件, 则系统将从爆炸式同步变为连续相变同步. 根据 这一理论认识, 文献[38]首次提出了一个比较实用 的控制爆炸式同步的方法. 其中心思想就是在系统 中引入部分反对者, 它们的作用就是抑制在系统中 形成大的同步集团. 基于对频率权重Kuramoto模型 的研究, 只需在系统中引进不超过 $8 \%$ 的反对者就可 以将爆炸式同步改变为连续相变 ${ }^{[38]}$.

\section{4 拥护者和反对者网络中的爆炸式同步}

\section{1 经典Kuramoto系统变例}

经典Kuramoto模型中, 全局耦合强度都是正的, 意味着振子之间的相互作用是“吸引”的, 这有利于 同步集团的形成. 然而, 在很多实际系统中, 个体之 间的相互作用可能是互相抑制的, 这种情况可以引 入负的耦合强度来描述. 例如, 神经网络中同时存 在兴奋性和抑制性的连接, 社会网络中存在负面的 相互影响. 为了描述这样的系统, 文献[15]研究了一 个经典Kuramoto模型的变例. 具体地讲, 就是在振 子相互作用中引入了负的耦合强度. 这样, 系统现 在由两种振子耦合而成, 那些耦合强度为正的振子 可称为“拥护者”, 而那些耦合强度为负的振子可称 为“反对者”. 更准确地讲, “拥护者”是从平均场中接 收正的相互作用, 而“反对者”则是从平均场中接收 负的相互作用. 系统的动力学方程为

$\dot{\theta}_{i}=\omega_{i}+\frac{\kappa_{i}}{N} \sum_{j=1}^{N} \sin \left(\theta_{j}-\theta_{i}\right), \quad i=1,2, \ldots, N$.

这里耦合强度 $\kappa_{i}$ 可取正或负, 本征频率满足单峰洛 伦兹分布(见表1). 为了简化问题和便于理论处理, 文献[15]假设 $\kappa_{i}$ 只取两个值: $\kappa_{1}<0$ 和 $\kappa_{2}>0$, 分别对 应反对者和拥护者. 定义拥护者的比例为 $P$, 图 8 给 出了系统的相变图. 如图8所示, 系统一开始时所振 子全部为反对者 $(P=0)$. 然后随机选取反对者变为 拥护者, 随着拥护者比例的增加, 系统从非协同态直 接跳变到 $\pi$ 态.

\section{2 耦合强度和频率关联}

文献[15]中反对者变为拥护者时, 反对者振子 是随机选取的. 稍后, 文献 $[39,40]$ 进一步考虑了 耦合强度与振子本征频率的关联. 具体地讲, 文 献 $[39,40]$ 研究了反对者变为拥护者时的 3 种典型策 略: (1) 随机选取; (2) 根据本征频率绝对值从大到 小选取; (3) 根据本征频率绝对值从小到大选取. 文 献[40]系统地研究了这3种情况下的同步相变, 并运 用线性稳定性分析和平均场理论求解出了相变点. 研究表明, 系统可能存在的稳态有非协同态、 $\pi$ 态、 
两种行波态和Bellerophon态, 并且非协同态是中性 稳定的. 随着拥护者比例 $P$ 的变化, 根据耦合强度 和本征频率的不同关联, 系统会表现出非常丰富的 相变行为, 图9给出了一个代表性的例子(详情见文 献[40]中的图2).

此外, 文献[41]系统地研究了频率权重耦合 的拥护者和反对者网络的同步相变. 考虑与文 献[40]相同的3种策略, 文献[41]运用线性稳定性分 析和平均场理论, 给出了系统所有可能出现的稳 态、从非协同态到同步态的相变途径以及相变点的 理论解, 并通过大量的数值模拟进行了验证.

\section{Bellerophon态}

如图5所示, 经典的爆炸式同步中, 向前相变 点 $\kappa_{\mathrm{f}}$ 一般大于向后相变点 $\kappa_{\mathrm{b}}$, 即 $\kappa_{\mathrm{f}}>\kappa_{\mathrm{b}}$. 例如在洛伦 兹分布下, 频率权重Kuramoto模型中的爆炸式同步 两个相变点分别为 $\kappa_{\mathrm{f}}=4$ 和 $\kappa_{\mathrm{b}}=2$. 然而深入的研究 表明, 这种情况并非一成不变. 在系统的某些参数 改变时, 向前相变点 $\kappa_{\mathrm{f}}$ 甚至可能小于向后相变点 $\kappa_{\mathrm{b}}$, 即 $\kappa_{\mathrm{f}}<\kappa_{\mathrm{b}}$. 此时在 $\kappa_{\mathrm{f}}<\kappa<\kappa_{\mathrm{b}}$ 的耦合强度区间, 系统

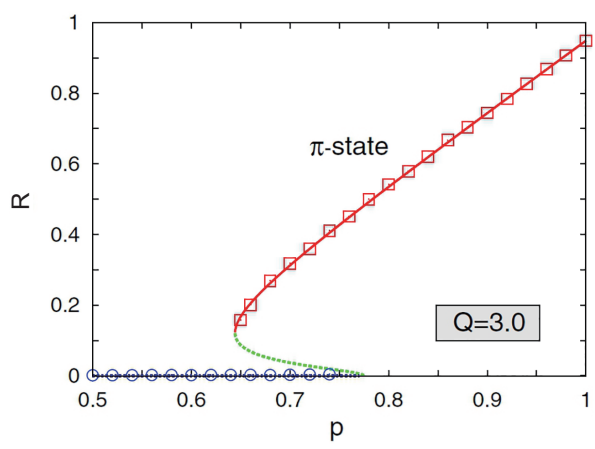

图 8 (网络版彩图)拥护者和反对者相振子耦合网络中的爆 炸式同步. 圆圈和正方形符号表示数值计算得到的序参量, 线表示理论预测. 其中实线表示稳定协同态, 点线表示不稳 定 $\pi$ 态. $N=25600, \kappa_{1}=-3, \kappa_{2}=1, Q=\left|\kappa_{1} / \kappa_{2}\right|=3$. 改编自参 考文献[15], 版权归美国物理学会所有

Figure 8 (Color online) Explosive synchronization in networked conformists and contrarians. Circles and squares are order parameters computed by numerical integration while the lines are theoretical predictions. Among them, solid lines denote stable states and the dotted line denotes unstable $\pi$ state. $N=25600, \kappa_{1}=-3, \kappa_{2}=1$, and $Q=\left|\kappa_{1} / \kappa_{2}\right|=3$. Adapted with permission from ref. [15]. Copyrighted by the American Physical Society.

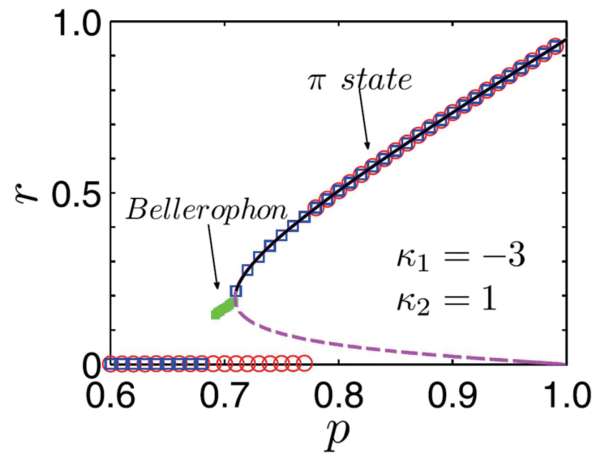

图 9 (网络版彩图)拥护者和反对者耦合网络中的爆炸式同 步, 这个例子是根据本征频率绝对值从大到小选取反对者, 将其变成拥护者. 符号为数值模拟结果, 线为理论预言. 圆圈 为向前相变, 方块为向后相变; 实线为稳定解, 虚线为不稳定 解. 频率分布为单峰洛伦兹分布

Figure 9 (Color online) Explosive synchronization in networked conformists and contrarians. In this example, the contrarians are flipped into the conformists according to their absolute magnitude of natural frequencies. Marks are results of numerical simulation and lines are theoretical prediction. Circles and squares denote the forward and the backward transitions, respectively, and the solid line and dashed line denote stable and unstable stationary states, respectively. The unimodal Lorentzian frequency distribution is adopted.

将形成一种协同性和异质性并存的高阶协同态. 这一节我们将详细介绍和刻画这种高阶同步态, 即Bellerophon态.

耦合振子系统的同步研究主要关注三大类的问 题: 一是相变点, 包括相变发生的形式(一级相变还 是二级相变)、相变点附近的分岔以及相变点的解 析求解等; 二是系统形成的协同态的特征和性质、 理论刻画和预言等; 三是系统的动力学与网络之间 如何相互作用和影响. 这些问题中, 协同态的研究处 于核心地位.

回顾Kuramoto一类模型过去四十多年的研 究(其他耦合振子系统类似), 已经发现的典型协 同态包括以下 5 种.

(1) 协同态. 这是耦合振子系统中最常见的宏观 有序态 ${ }^{[6,7]}$. 一般地, 随着耦合强度的增大, 系统中 有部分振子开始形成宏观上的协同运动, 例如锁相 或锁频. 这部分同步的振子会导致系统的宏观序参 量(平均场)大于零, 从而进一步驯服更多的游离振 子加入同步集团.

(2) $\pi$ 态. 这是拥护者与反对者耦合的Kuramoto 
模型中以及二阶耦合的Kuramoto模型中发现的一 种协同态, 其性质与(1)中描述的协同态类似 ${ }^{[15]}$. 只 不过这里系统中的部分拥护者和部分反对者各自形 成一个同步集团, 并且这两个集团之间的相位差保 持为 $\pi$.

(3) 行波态. 这也是一种常见的协同态, 特别是 当系统中振子的本征频率分布不对称, 或系统中耦 合强度不对称的时候容易形成 ${ }^{[35]}$. 这时系统形成 的同步集团以一定的频率(角速度)在单位圆上旋转, 其整体表现就相当于一个大振子.

(4) 驻波态. 在这种协同态中, 相振子形成两个 集团(一般是大小相等), 并以相同大小的角速度在 单位圆上旋转, 但方向相反. 整体上看, 两个集团等 效为两个大振子, 它们的频率大小相等, 但是一个为 正, 另一个为负.

(5) Chimera态(简称 $C$ 态). Chimera是希腊神话 中半人半兽的怪物, 以此命名的协同态是一种非常 有趣的态, 故又称为“奇妙态”或“奇异态”. 它神奇在 什么地方呢? 表面上看, $\mathrm{C}$ 态中同步集团和游离振 子共存, 似乎与普通的协同态没有什么区别, 但实际 上C态的神奇之处就在于它竟然形成于振子全同、 耦合对称的系统之中! 实际上它是振子初始条件 对称破缺而形成的特殊的宏观有序态. C态的研究 得到了持续的关注, 它有助于揭示海豚和一些鸟类 奇特的半脑睡眠现象(一半大脑在休息, 另一半在工 作).

以上同步系统中常见的协同态可以分成两 类: 平稳(Stationary)和非平稳(Non-Stationary). 根据 第3.2节引入的几率密度函数, 它描述振子在相空 间(单位圆上)中的分布. 如果协同态的几率密度函 数不随时间变化, 则称为平稳态; 否则就是非平稳 态. 在上述的 5 种协同态中, 协同态 $\pi$ 态和行波态 为平稳协同态(行波态可放在与同步振子集团旋转 频率相同的旋转参考系中观察); 驻波态为非平稳协 同态; 而 $\mathrm{C}$ 态既有平稳的, 又有非平稳的. 平稳协同 态可认为是低阶的同步态, 其性质已经得到了比较 充分的研究. 而对于非平稳协同态, 由于这类态具有 时变性质, 可认为是高阶的同步态, 不仅目前发现的 不多,而且其理论描述和研究非常困难.

2016年, 文献[32]报道了一种新的高阶协同
态—Bellerophon态(简称B态). 这种协同态首先在 双峰洛伦兹分布下(表1)的频率权重模型方程(10)发 现. 文献[32]从理论上推导出了系统同步的相变 点, 包括向前和向后相变点的解析形式. 当参 数 $\omega_{0} / \Delta$ 增大时, 双峰洛伦兹分布的两个峰之间的 距离逐渐变大, 频率分布从单峰转变为双峰(转变点 在 $\left.\omega_{0} / \Delta=\sqrt{3} / 3\right)$. 如图6所示, 在此过程中, 向后相 变点始终保持不变 $\left(\kappa_{\mathrm{b}}=2\right)$, 但是向前相变点逐渐 减小. 直到 $\omega_{0} / \Delta>\sqrt{3}$ 时, 向前相变点 $\kappa_{\mathrm{f}}<\kappa_{\mathrm{b}}$ ! 这一 过程对应的相变图像如图10所示: 随着 $\omega_{0} / \Delta$ 的增大, 向前相变点不断减小, 导致磁滞区逐渐缩小, 而系 统的同步相变也由一级相变逐渐转化为二级相变. 当 $\kappa_{\mathrm{f}}<\kappa_{\mathrm{b}}$ 时, 如图10(e)和(f)所示, 此时系统通过连续 相变进入协同态. 以图10(f)为例, 研究发现当耦合 强度比较小时 $\left(\kappa<\kappa_{\mathrm{f}}\right)$, 系统处于非协同态; 而当耦 合强度足够大时 $\left(\kappa>\kappa_{\mathrm{b}}\right)$, 系统处于协同态, 同步集 团的振子处于瞬时频率锁频状态. 而在中等耦合强 度区域 $\left(\kappa_{\mathrm{f}}<\kappa<\kappa_{\mathrm{b}}\right)$, 系统将处于一种量子化的、多 集团的时变协同态, 即 $\mathrm{B}$ 态.

图11给出了一个B态的例子, 对其进行了详细 的刻画. 该态对应于图10(f)中的 $\mathrm{B}$ 点 $(\kappa=1.60)$. 如
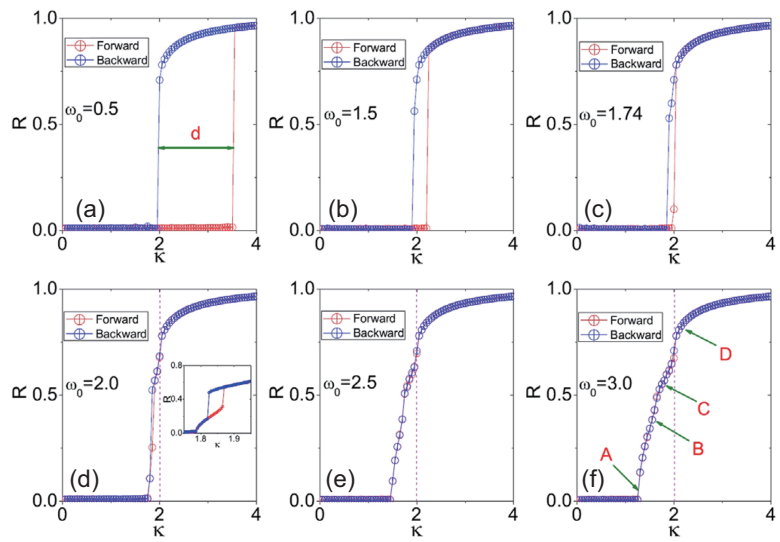

图 10 (网络版彩图)双峰洛伦兹频率分布下, 频率权 重Kuramoto模型中的同步相变, $\Delta=1$. 图示为序参量 $R$ 随 耦合强度 $\kappa$ 的变化. 当 $\omega_{0}$ 增大时, 系统由一级相变转化为二级 相变

Figure 10 (Color online) Synchronization transitions of frequencyweighted Kuramoto model with bimodal Lorentzian distribution, characterized by order parameter $R$ vs coupling strength $\kappa, \Delta=1$. As $\omega_{0}$ increases, the synchronization transition converts from the first to the second type. 

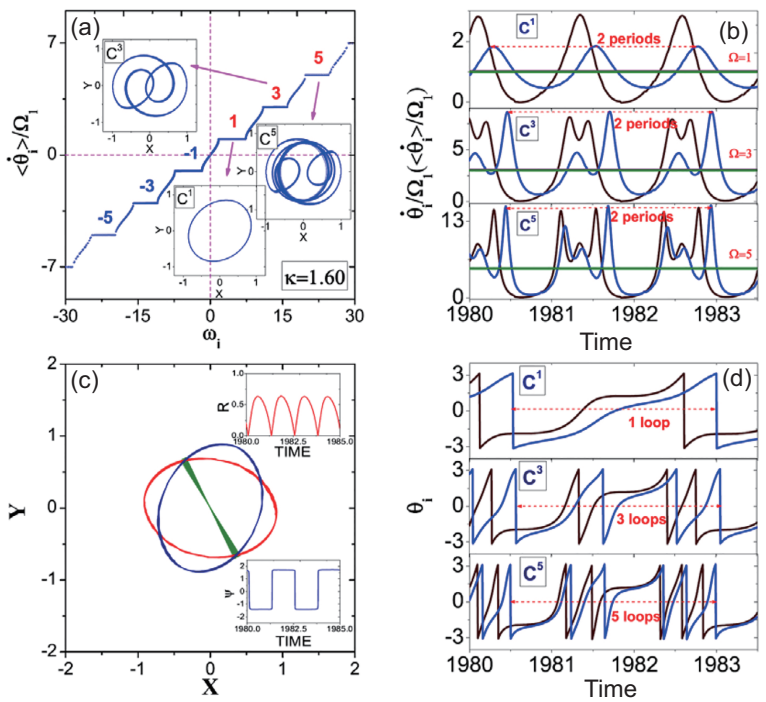

图 11 (网络版彩图)一个典型的B态. (a) 协同集团的平均频 率, 对应于主频 $\Omega_{1}$ 的奇数倍. (b) 两个协同振子的瞬时角速 度, 它们是随机地从集团 $C^{1}$ (顶行), $C^{3}$ (中间行)和 $C^{5}$ (底行)中 选取. 直线表示平均角速度. (c) 频率为正的振子的序参量(蓝 色椭圆)、频率为负的振子的序参量(红色椭圆)以及全部振 子的序参量(绿线). 插图为全局序参量 $R(t)$ 和 $\Psi(t)$ 的时间序列, 呈振荡演化. (d) 对应于(b)的瞬时相位演化

Figure 11 (Color online) A typical B state. (a) The average speeds of the coherent clusters. They correspond to odd-numbered multiples of the fundamental frequency $\Omega_{1}$. (b) Time series of the instantaneous speeds of the clustered oscillators. In the panel, two sample oscillators are arbitrarily chosen from clusters $C^{1}$ (top), $C^{3}$ (middle), and $C^{5}$ (bottom). The straight lines mark the average speed. (c) Order parameters for all oscillators with positive (blue oval) and negative (red oval) frequency, and order parameter for all oscillators (green lines). The insets are the time series of the global order parameter $R(t)$ and $\Psi(t)$, which are typically oscillatory. (d) Time series of the instantaneous phases corresponding to (b).

图11(a)所示, B态有很多成对、对称的协同集团, 其 平均频率(角速度)呈阶梯状. 更为有趣的是, 所有的 频率台阶都是基频(最小频率)的奇数倍, 即量子化. 这样平均而言, 在一个周期 $\left(2 \pi / \Omega_{1}\right)$ 内, 第一协同集 团的振子转动 1 圈, 第二协同集团的振子转动 3 圈, 以此类推, 如图11(d)所示. 图11(b)进一步揭示了B态 的主要特征, 即协同集团中的振子的瞬时频率并不 锁定, 但是它们的平均频率却是锁定的. 图11(c)显 示序参量呈周期振荡.

从上面的例子可以看出, B态中同一个协同集 团内的振子表现出非常复杂的行为, 协同性与异质
性并存, 远非传统意义上简单的锁频或锁相可以描 述, 它实际上是振子动力学的异质性与耦合导致的 协同性之间通过竞争获得的一种妥协状态. 打一个 比方, 普通的协同态就像是军人出操形成的整齐方 阵, 而 $\mathrm{B}$ 态就像是奥运会万米长跑中有时形成的运 动员集团, 同一团体内既有相互协作又有相互竞争, 因此同一集团内的运动员不是简单的完全同步, 而 是协同性与异质性的竞争与统一.

B态最先是在频率耦合权重Kuramoto模型中发 现的 ${ }^{[32,34]}$, 接着文献[42]进一步报道了一个具有阵 发性特征的B态, 如图12所示. 稍后, 在包含拥护者 和反对者的广义Kuramoto模型中也发现了这类同 步态 ${ }^{[40,41]}$, 并且此类模型中还发现了其他类型的高
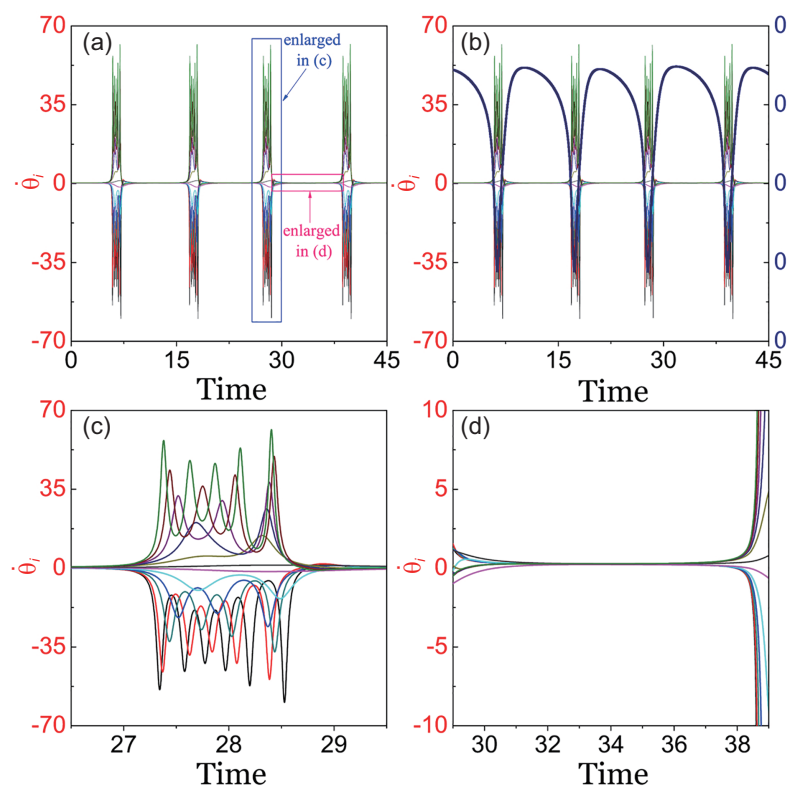

图 12 (网络版彩图)具有阵发性质的 $\mathrm{B}$ 态: 振子在大多数情 况下基本处于静止状态, 但在一系列小的时间窗口里面爆 发 (以较大的角速度旋转). (a) 振子的瞬时速度随时间的演 化. 这里在12 个同步集团中每一个集团里面随机选取一 个振子. (b) 振子的瞬时速度和系统序参量随时间的演化. (c)和(d)对应(a)图中的局部放大

Figure 12 (Color online) An intermittent B state: oscillators are almost resting during most time while burst (rotating with high speed) in successive short periods. (a) Evolution of the instantaneous speeds of sample oscillators. For better visualization, we only choose 12 clusters and sample one oscillator in each cluster. (b) Evolution of the instantaneous speeds and the order parameter. (c) and (d) Enlargement of the boxes in (a) respectively. 
阶协同态. 迄今为止, 已经在多种模型、多种 频率分布下都观察到了B态 ${ }^{[32,34,40-44]}$. 特别是文 献 $[43,44]$ 发现了双峰洛伦兹分布下经典Kuramoto模 型中也能出现 $\mathrm{B}$ 态. 这表明 $\mathrm{B}$ 态并非某一种特殊模型 或特殊频率分布导致的结果, 它很可能广泛存在于 耦合相振子系统之中, 因此非常值得进一步深入的 探索.

总结一下目前对 $\mathrm{B}$ 态的研究结果, 可以发现 $\mathrm{B}$ 态 是一种量子化的、多集团的时变协同态. 它出现在 本征频率不同、即非全同耦合振子系统中, 这一点 和 C态的根本不同. 这种协同态具有 3 个基本特征. (1) 量子性. 在这种同步态中, 振子可以形成具有不 同平均频率的协同集团, 它们和游离振子共存. 协 同集团振子的平均频率是某一个基频的倍数, 可以 是奇数倍、偶数倍或整数倍. (2) 时变性. 虽然每个 协同集团内振子的平均频率相同, 但它们的瞬时频 率(瞬时角速度)各不相同, 并且它们的瞬时频率表 现出高阶相关性, 并非简单的锁频. (3) 异质性. 同一 协同集团中不同振子的瞬时频率不仅是时变的, 而 且各不相同, 表现出高度的异质性.

从定性上看, 耦合振子系统中出现B态不难解 释. 在耦合强度较小的情况下, 平均场不足以驯服 具有频率差异的振子, 系统因此处于非协同态, 系统 的动力学维度最高, 此时单个振子具有最大的自由 度. 另一方面, 当耦合强度足够大时, 振子之间的相 互作用克服了其频率差异性, 系统因此处于完全协 同态, 振子之间的瞬时频率是锁定的, 整个同步集团 相当于一个大振子, 系统的动力学维度最低, 此时 单个振子的自由度最小. 当系统处于中间耦合强度 时, 此种耦合强度虽然尚不能形成比较强的完全协 同态, 但仍然可以使振子形成某种比较弱的关联, 就 像B态那样, 瞬时频率不能锁定, 但平均频率却被锁 定. 同一集团内的振子虽然具有一定的关联性(协同 性), 但仍然表现出相当的自由度(异质性). 定量上, 文献[45]应用圆映射理论揭示了频率权重模型中出 现奇数倍平均频率台阶的机制.

\section{6 多层网络中的爆炸式同步}

最早发现的爆炸式同步模型中都作了两个假
设: (1) 无标度网或星形网; (2) 度和频率正相关. 后 来频率权重模型放宽了第一个假设, 基本上不依 赖于网络拓扑结构. 但第二个条件能否放宽, 这是 一个值得深入的问题. 在文献[46]中, 我们研究了 两个共演化网络, 即同一套节点, 两套不同性质的 边 $G_{1}$ 和 $G_{2}$. 然后假设Kuramoto相振子通过 $G_{1}$ 相互连 接, 但是我们没有采用 $\omega_{i}=k_{1, i}$, 即度和频率相等的 假设, 而是假定 $\omega_{i}=k_{2, i}$, 这样 $\omega_{i}$ 只是 $k_{1, i}$ 的函数. 结果 表明, 在一定条件下系统可以呈现爆炸式同步. 这 样, 文献[46]的工作将爆炸式同步出现的强关联条 件 $\left(\omega_{i}=k_{i}\right)$ 放宽到弱条件 $\left[\omega_{i}=f\left(k_{i}\right)\right]$.

2015年, 文献[47]进一步研究了双层网络上的 自适应同步, 发现在引入自适应的条件下, 系统 可以出现爆炸式同步相变, 完全不需要度和频率 正相关这一条件. 文献[47]先研究了单个ER网络上 的Kuramoto模型. 假设有比例为 $f$ 的振子采用自适 应演化, 对其中任何一个振子 $i$, 它与平均场的耦合 强度正比于它的局域序参量 $r_{i}$. 结果表明, 当 $f$ 足够 大时, 系统可以出现爆炸式同步.

接着, 文献[47]研究了双层网络上的自适应同 步. 如图13所示, 文献[47]中构造了两个具有相同节 点的网络, 但这两个网络的拓扑结构和振子的本 征频率不同. 选取比例为 $f$ 的振子为自适应演化振 子(图13中蓝色振子), 它们可以在两个网络之间相 互作用, 即第一层网络的振子耦合强度正比于与其 相连的第二层网络上对应振子的局域序参量, 反之 亦然. 系统的动力学方程为

$\dot{\theta}_{i, 1}=\omega_{i, 1}+\kappa \alpha_{i, 1} \sum_{j=1}^{k_{i, 1}} \sin \left(\theta_{j, 1}-\theta_{i, 1}\right)$,

$\dot{\theta}_{i, 2}=\omega_{i, 2}+\kappa \alpha_{i, 2} \sum_{j=1}^{k_{i, 2}} \sin \left(\theta_{j, 2}-\theta_{i, 2}\right)$,

式中, 下标 1 和 2 表示网络编号. 如图13所示, 这里的 中心思想是第一层网络的自适应振子受到第二层对 应振子的序参量调制, 反之亦然, 即对自适应振子 $i$, $\alpha_{i, 1}=r_{i, 2}, \alpha_{i, 2}=r_{i, 1}$; 对于非自适应振子(比例为 $1-f$ ), $\alpha_{i, 1}=1, \alpha_{i, 2}=1 . f=1$ 时的结果如图14所示, 在两 个网络拓扑结构和振子本征频率的多种组合下, 系 统普遍出现爆炸式同步相变. 之后, 文献[48]进一步 研究了 $f<1$ 的情形, 得出了爆炸式同步和经典同 
步共存的结论; 文献[49]研究了多层网上KuramotoSakugchi模型中的爆发式同步. 另外, 文献[50]利用 序参量自适应控制的思想研究了爆炸式同步与奇异 态的联系; 文献[51]研究了自适应网络中的爆炸式 同步.

\section{7 爆炸式同步的实验验证}

2012年, 爆炸式同步首次在电路系统中得到 实验验证. 文献[14]首先研究了无标度网络上 的Rössler振子的同步. 在假设振子的频率与度正 相关的前提下, 观察到了系统能出现爆炸式相同 步. 进一步, 文献[14]设计了一个电路, 由6个处于混 沌状态的Rössler振子构成一个星形网络: 中心1个, 周边 5 个, 这样形成的网络具有最大的异质性, 如 图15所示. 实验结果表明, 在合适的参数下, 系统能 表现出爆炸式同步相变, 向前和向后相变形成明显 的磁滞区, 如图16所示.

2015年, Kumar等人 ${ }^{[52]}$ 在耦合化学-机械系统中 观察到了爆炸式同步.他们研究是一类称为 $\mathrm{MBH}$

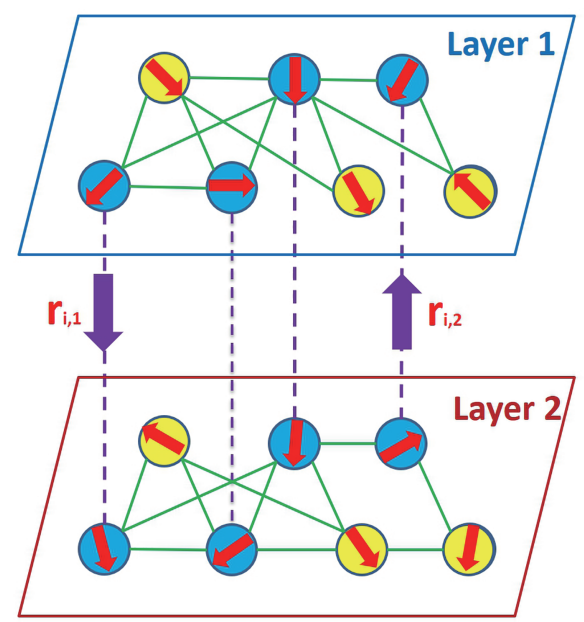

图 13 (网络版彩图)双层网络振子自适应耦合示意图. 每一 层振子数目相同, 但是网络结构不同. 对于自适应演化的振 子, 其有效耦合强度正比于另一层网络对应振子的局域序 参量

Figure 13 (Color online) Schematic plot of the adaptive coupling in two-layer networks. Each layer has the same number of nodes, but different topology. For adaptive oscillators (bule circles), their effective coupling strength depends on the local order parameter of its counterpart in the other layer.
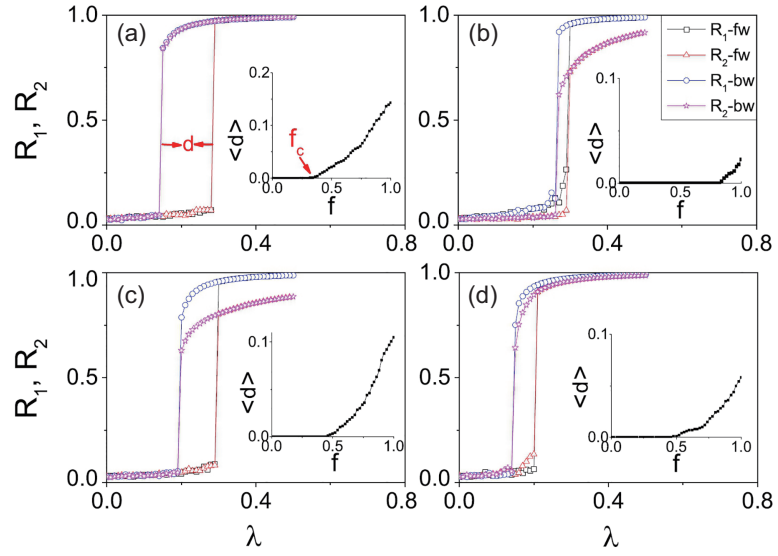

图 14 (网络版彩图)自适应双层网络的同步相变. $N=1000$, $f=1 . R_{1}\left(R_{2}\right)$ 对应第 $\mathrm{I}(\mathrm{II})$ 层的序参量, 插图表示磁滞区宽度对 比例参数 $f$ 的依赖关系(10次平均). 第 $\mathrm{I}$ 层固定为ER网络, 平 均度为 12 , 振子频率为 $[-1,1]$ 区间的均匀分布. 第II层网络的 参数如下: (a) ER网络, 平均度为 12 , 振子频率为 $[-1,1]$ 区间 的均匀分布; (b) ER 网络, 平均度为 6 , 振子频率分布与 (a)相 同; (c) 网络与(a)相同, 但振子频率为洛伦兹分布; (d) BA 网 络, 平均度为 12 , 振子频率分布与(a)相同

Figure 14 (Color online) Synchronization transitions in two-layer networks. $N=1000$ and $f=1 . R_{1}\left(R_{2}\right)$ is the order parameter of the layer I (II) and the insets show the corresponding dependence of $<d>$ on $f$ for ten realizations. Layer I is fixed as a random ER network with average degree $\left\langle k_{1}>=12\right.$ and having a random homogeneous distribution of frequencies in the range $[-1,1]$. Layer II has different specifications, as follows: (a) it is another ER network with $<k_{2}>=12$, and the frequency distribution is homogeneous in the range $[-1,1]$; (b) it is an ER network with $\left\langle k_{2}>=6\right.$ and the frequency distribution is the same as in (a); (c) the same as in (a), but the frequency distribution is now Lorentzian; (d) a BA network with $\left\langle k_{2}>=12\right.$ and the frequency distribution is the same as in (a).

(Mercury Beating-Heart)的振子网络. 如图17所示, 4 个振子形成星形网络, 中间 1 个, 周边 3 个. 在实验 中, 他们设法调节振子的频率, 使其与度成正比, 这样就满足了频率和度相关的条件. 实验结果如 图18所示. 当频率和度相关的条件满足时, 系统表 现出爆炸式同步, 向前和向后相变形成明显的磁滞 区; 而当频率和度相关的条件不满足时, 系统表现出 连续相变, 向前和向后相变曲线基本重合.

\section{8 结束语}

自2011年以来, 爆炸式同步迅速成为了复杂系 统研究前沿的热点, 吸引了大批学者的关注. 目前, 


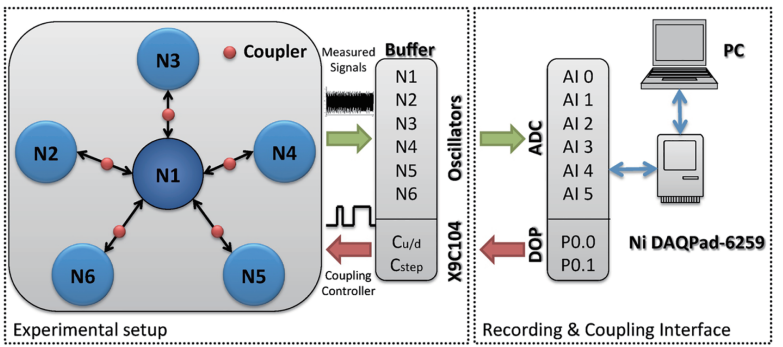

图 15 (网络版彩图)验证爆炸式同步的实验装置图: 由6个Rössler电路振子形成星形网络. 改编自参考文献[14], 版权归美国物理学会所有

Figure 15 (Color online) Schematic plot of the experimental setup verifying explosive synchronization, where six Rössler circuits (blue nodes) are bidirectionally connected to form a star configuration. Adapted with permission from ref. [14]. Copyrighted by the American Physical Society.

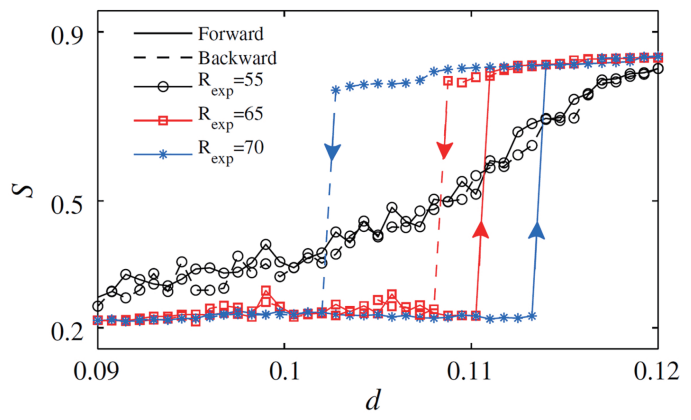

图 16 (网络版彩图)序参量 $r$ 随耦合强度 $\alpha$ 的变化对系统同步 相变的刻画. $R_{\exp }$ 是电路的内阻. 当 $R_{\exp }=55$ 时, 同步相变为 二阶, 而当 $R_{\exp }=65$ 或 70 时, 同步相变为一阶. 改编自参考文 献[14], 版权归美国物理学会所有

Figure 16 (Color online) Synchronizaton transitions characterized by order parameter $r$ vs coupling strength $\kappa . R_{\exp }$ is the internal resistance of the circuit. When $R_{\exp }=55$, the second-order transition occurs, while when $R_{\exp }=65$ or 70, the first-order transitions occur. Adapted with permission from ref. [14]. Copyrighted by the American Physical Society.

在包括频率和度关联振子系统、频率权重Kuramoto 模型、拥护者和反对者耦合相振子系统、多层网络 自适应系统等方面已经开展了深入的研究. 这些工 作开拓了新的方向, 极大地丰富了同步理论的研究 范畴, 不仅加深了我们对于复杂系统协同动力学行 为的理解, 而且为将来相关领域的应用打下了基础.

虽然已经取得了阶段性的成果, 但对于爆炸式 同步这样一个具有挑战性的新课题而言, 目前的研 究尚在起步阶段, 这里面还有许多非常重要的理论 和应用问题亟待研究, 主要包括:

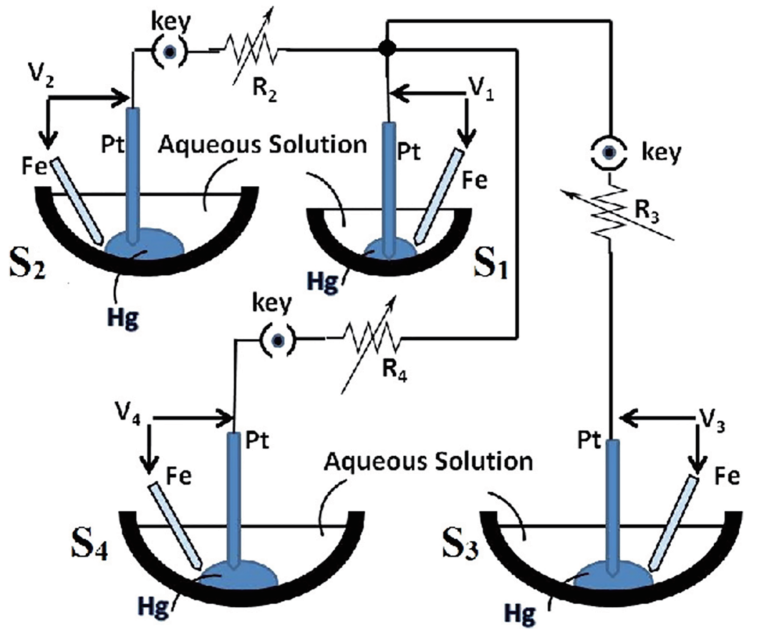

图 17 (网络版彩图)4个MBH振子形成星形网络的实验示意 图. 改编自参考文献[52], 版权归美国物理学会所有

Figure 17 (Color online) Schematic plot of the experimental setup of 4 MBH oscillators connected in a star network. Adapted with permission from ref. [52]. Copyrighted by the American Physical Society.

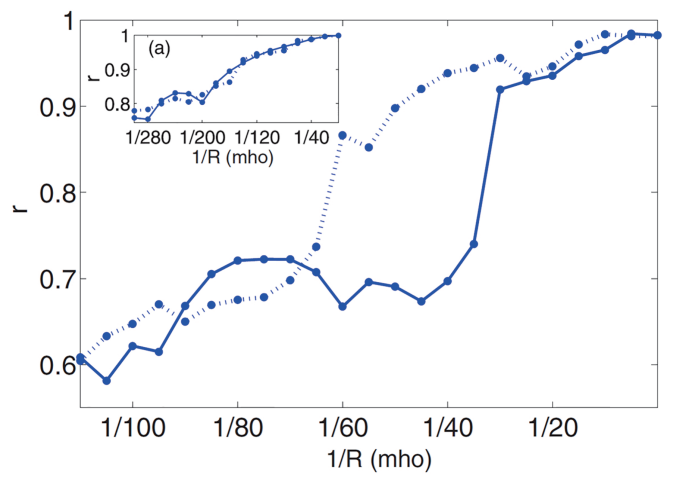

图 18 (网络版彩图)耦合 $\mathrm{MBH}$ 振子在度和频率正相关条件 下形成爆炸式同步. $r$ 是序参量, $1 / R$ 是耦合强度. 与之相反, 当振子频率基本相同时, 插图显示系统形成连续相变. 改编 自参考文献[52], 版权归美国物理学会所有

Figure 18 (Color online) Explosive synchronization observed in coupled MBH oscillators when their degrees are positively correlated with the frequencies. $r$ is the order parameter and $1 / R$ is the coupling strength. On the contrary, continuous transition is shown in the inset when the oscillators have almost the same frequencies. Adapted with permission from ref. [52]. Copyrighted by the American Physical Society.

(1) 模型. 当前能产生爆炸式同步的系统主要是 广义Kuramoto模型, 也就是耦合相振子模型, 它们不 涉及振幅的动力学行为. 我们知道, 更一般和更实 际的情况是, 振子的动力学行为由振幅和相位共同 描述. 之前对耦合混沌振子的同步化研究已经表明: 
系统存在完全同步、广义同步和相同步等不同层次 的同步化行为. 其中相同步只是一种比较弱的情形, 对于比较强的完全同步和广义同步, 振子间的振幅 将发生强关联, 在这种情况下耦合振子系统是否也 有类似的爆炸式同步化行为? 目前这方面的研究刚 刚开展, 已经在含振幅动力学的系统中观察到了爆 炸式同步化现象 ${ }^{[14,53,54]}$, 无疑它们是下一步理论研 究的重要基本问题.

(2) 机制. 为何以前耦合振子系统大都表 现出连续的同步化相变, 而最近发现的一些广 义Kuramoto系统却能表现出爆炸式同步化行为? 刚开始, 研究发现振子的动力学与网络节点度的正 相关可能是产生爆炸式同步化的条件 ${ }^{[13]}$; 后来又 发现: 只要网络部分中心节点的度与振子频率正 相关就可以产生爆炸式同步化 ${ }^{[55]}$; 在复GinzburgLandau方程中, 这种相关性甚至可以由网络与动力 学的相互作用自组织出来 ${ }^{[24]}$; 而在序参量自适应耦 合模型中 ${ }^{[38]}$, 爆炸式同步化的出现已经不再依赖于 振子频率与网络节点度之间的正相关. 爆炸式同步
化现象下面的物理机制是什么? 什么因素在这里起 关键性的作用? 这些都是爆炸式同步的核心问题, 非常值得深入研究.

(3) 高阶协同态. B态的发现不仅揭示了耦合相 振子系统中可以表现出既协同又具有相对自由度 的高阶同步行为, 而且为进一步理论和实验研究提 供了重要的思路. 例如, 生命科学的实验已经揭示: 简单的、完全的同步态往往导致系统出现病态, 如 心肌细胞同步形成规则的螺旋波可能是房颤的先 兆; 大脑神经元在极短时间内的强同步放电会导致 癫㾁病发作等. 因此, 我们不仅要研究简单的、平 稳的协同态, 更应该加强对非平稳、时变的高阶协 同态的研究. 然而对高阶协同态进行刻画和预测是 极富创新性和挑战性的理论工作. 现有的理论方法, 如平均场自洽方法、线性稳定性分析、振幅方程理 论、Watanabe-Strogatz变换方法和Ott-Antonsen拟设 等不足以分析和处理高阶协同态, 这里亟需发展新 的方法来解决高阶协同态的稳定性、相变点等基本 问题, 并在此基础上揭示其形成机制. 李薛、张加蒙.

\section{参考文献}

1 Pikovsky A, Rosenblum M, Kurths J. Synchronization: A Universal Concept in Nonlinear Sciences. Cambridge: Cambridge University Press, 2003

2 Boccaletti S, Pisarchik A N, del Genio C I, et al. Synchronization: From Coupled Systems to Complex Networks. Cambridge: Cambridge University Press, 2018

3 Hu G, Xiao J H, Zheng J H. Chaos Control (in Chinese). Shanghai: Shanghai Science and Technology Education Press, 2000 [胡岗, 萧井华, 郑 志刚. 混沌控制. 上海: 上海科技教育出版社, 2000]

4 Boccaletti S, Latora V, Moreno Y, et al. Complex networks: Structure and dynamics. Phys Rep, 2006, 424: 175-308

5 He D R, Liu Z H, Wang B H. Complex Systems and Networks (in Chinese). Beijing: Higher Education Press, 2009 [何大韧, 刘宗华, 汪秉宏. 复 杂系统与复杂网络. 北京: 高等教育出版社, 2009]

6 Kuramoto Y. Chemical Oscillations, Waves, and Turbulence. Berlin: Springer, 1984

7 Strogatz S H. From Kuramoto to Crawford: Exploring the onset of synchronization in populations of coupled oscillators. Phys D-Nonlinear Phenom, 2000, 143: 1-20

8 Pecora L M, Carroll T L. Master stability functions for synchronized coupled systems. Phys Rev Lett, 1998, 80: 2109-2112

9 Rodrigues F A, Peron T K D, Ji P, et al. The Kuramoto model in complex networks. Phys Rep, 2016, 610: 1-98

10 Yeung M K S, Strogatz S H. Time delay in the Kuramoto model of coupled oscillators. Phys Rev Lett, 1999, 82: 648-651

11 Pazó D. Thermodynamic limit of the first-order phase transition in the Kuramoto model. Phys Rev E, 2005, 72: 046211

12 Martens E A, Barreto E, Strogatz S H, et al. Exact results for the Kuramoto model with a bimodal frequency distribution. Phys Rev E, 2009, 79: 026204

13 Gómez-Gardeñes J, Gómez S, Arenas A, et al. Explosive synchronization transitions in scale-free networks. Phys Rev Lett, 2011, 106: 128701 
14 Leyva I, Sevilla-Escoboza R, Buldú J M, et al. Explosive first-order transition to synchrony in networked chaotic oscillators. Phys Rev Lett, 2012, 108: 168702

15 Hong H, Strogatz S H. Kuramoto model of coupled oscillators with positive and negative coupling parameters: An example of conformist and contrarian oscillators. Phys Rev Lett, 2011, 106: 054102

16 Boccaletti S, Almendral J A, Guan S, et al. Explosive transitions in complex networks' structure and dynamics: Percolation and synchronization. Phys Rep, 2016, 660: 1-94

17 Achlioptas D, D’Souza R M, Spencer J. Explosive percolation in random networks. Science, 2009, 323: 1453-1455

18 Haken H, Ling F H (Translator). Synergetics: The Science of Structure (in Chinese). Shanghai: Shanghai Translation Publishing House, 2013 [赫 尔曼.哈肯, 著. 凌复华, 译. 协同学: 大自然构成的奥秘.上海: 上海译文出版社, 2013]

19 Zou Y, Pereira T, Small M, et al. Basin of attraction determines hysteresis in explosive synchronization. Phys Rev Lett, 2014, 112: 114102

20 Pereira T, Eroglu D, Bagci G B, et al. Connectivity-driven coherence in complex networks. Phys Rev Lett, 2013, 110: 234103

21 Vlasov V, Zou Y, Pereira T. Explosive synchronization is discontinuous. Phys Rev E, 2015, 92: 012904

$22 \mathrm{Xu}$ C, Gao J, Sun Y, et al. Explosive or continuous: Incoherent state determines the route to synchronization. Sci Rep, 2015, 5: 12039

23 Coutinho B C, Goltsev A V, Dorogovtsev S N, et al. Kuramoto model with frequency-degree correlations on complex networks. Phys Rev E, 2013, 87: 032106

24 Chen Y, Cao Z, Wang S, et al. Self-organized correlations lead to explosive synchronization. Phys Rev E, 2015, 91 : 022810

25 Zhang X, Hu X, Kurths J, et al. Explosive synchronization in a general complex network. Phys Rev E, 2013, 88: 010802

$26 \mathrm{Hu} \mathrm{X}$, Boccaletti S, Huang W, et al. Exact solution for first-order synchronization transition in a generalized Kuramoto model. Sci Rep, 2015, 4: 7262

27 Xu C, Sun Y, Gao J, et al. Synchronization of phase oscillators with frequency-weighted coupling. Sci Rep, 2016, 6: 21926

28 Qiu T, Zhang Y, Liu J, et al. Landau damping effects in the synchronization of conformist and contrarian oscillators. Sci Rep, 2016, 5: 18235

29 Xu C, Gao J, Xiang H, et al. Dynamics of phase oscillators with generalized frequency-weighted coupling. Phys Rev E, 2016, 94 : 062204

30 Lotfi N, Rodrigues F A, Darooneh A H. The role of community structure on the nature of explosive synchronization. Chaos, 2018, 28: 033102

31 Wu H, Kang L, Liu Z, et al. Exact explosive synchronization transitions in Kuramoto oscillators with time-delayed coupling. Sci Rep, 2018, 8: 15521

32 Bi H, Hu X, Boccaletti S, et al. Coexistence of quantized, time dependent, clusters in globally coupled oscillators. Phys Rev Lett, 2016, 117: 204101

33 Pietras B, Deschle N, Daffertshofer A. First-order phase transitions in the Kuramoto model with compact bimodal frequency distributions. Phys Rev E, 2018, 98: 062219

34 Bi H J, Li Y, Zhou L, et al. Nontrivial standing wave state in frequency-weighted Kuramoto model. Front Phys, 2017, 12: 126801

35 Zhou W, Chen L, Bi H, et al. Explosive synchronization with asymmetric frequency distribution. Phys Rev E, 2015, $92: 012812$

36 Zhang X, Zou Y, Boccaletti S, et al. Explosive synchronization as a process of explosive percolation in dynamical phase space. Sci Rep, 2015, 4: 5200

37 Kim M, Mashour G A, Moraes S B, et al. Functional and topological conditions for explosive synchronization develop in human brain networks with the onset of anesthetic-induced unconsciousness. Front Comput Neurosci, 2016, 10: 1

38 Zhang X, Guan S, Zou Y, et al. Suppressing explosive synchronization by contrarians. Europhys Lett, 2016, 113: 28005

39 Yuan D, Zhang M, Yang J. Dynamics of the Kuramoto model in the presence of correlation between distributions of frequencies and coupling strengths. Phys Rev E, 2014, 89: 012910

40 Qiu T, Boccaletti S, Bonamassa I, et al. Synchronization and Bellerophon states in conformist and contrarian oscillators. Sci Rep, 2016, 6: 36713

41 Qiu T, Bonamassa I, Boccaletti S, et al. Rhythmic synchronization and hybrid collective states of globally coupled oscillators. Sci Rep, 2018, 8: 12950

42 Zhou W, Zou Y, Zhou J, et al. Intermittent Bellerophon state in frequency-weighted Kuramoto model. Chaos, 2016, $26: 123117$

43 Li X, Qiu T, Boccaletti S, et al. Synchronization clusters emerge as the result of a global coupling among classical phase oscillators. New J Phys, 2019, 21: 053002

$44 \mathrm{Li} \mathrm{X}$, Zhang J, Zou Y, et al. Clustering and Bellerophon state in Kuramoto model with second-order coupling. Chaos, 2019, 29: 043102

45 Xu C, Boccaletti S, Guan S, et al. Origin of Bellerophon states in globally coupled phase oscillators. Phys Rev E, 2018, 98 : 050202

46 Su G, Ruan Z, Guan S, et al. Explosive synchronization on co-evolving networks. Europhys Lett, 2013, 103: 48004

47 Zhang X, Boccaletti S, Guan S, et al. Explosive synchronization in adaptive and multilayer networks. Phys Rev Lett, 2015, 114: 038701

48 Danziger M M, Moskalenko O I, Kurkin S A, et al. Explosive synchronization coexists with classical synchronization in the Kuramoto model. Chaos, 2016, 26: 065307 
51 Avalos-Gaytán V, Almendral J A, Leyva I, et al. Emergent explosive synchronization in adaptive complex networks. Phys Rev E, 2018, 97: 042301

52 Kumar P, Verma D K, Parmananda P, et al. Experimental evidence of explosive synchronization in mercury beating-heart oscillators. Phys Rev E, 2015, 91: 062909

53 Bi H, Hu X, Zhang X, et al. Explosive oscillation death in coupled Stuart-Landau oscillators. Europhys Lett, 2014, 108: 50003

54 Zhang J M, Li X, Zou Y, et al. Novel transition and Bellerophon state in coupled Stuart-Landau oscillators. Front Phys, 2019, 14: 33603

55 Pinto R S, Saa A. Explosive synchronization with partial degree-frequency correlation. Phys Rev E, 2015, 91: 022818

\title{
Explosive synchronization in complex networks
}

\author{
GUAN ShuGuang* \\ School of Physics and Material Science, East China Normal University, Shanghai 200241, China
}

\begin{abstract}
Synchronization in coupled oscillators is one of the most important filed in statistical physics and complex systems. In the past a few decades, it is shown that most synchronization transitions are continuous (second-order), i.e., the order parameter increases continuously after the transition point. However, recently it has been revealed that in certain systems of coupled oscillators, synchronization could be explosive (first-order), where the order parameter suddenly jumps after the transition and a hysteresis loop exists due to the irreversibility between the forward and backward transitions. These findings have opened a new area since the explosive synchronization is essentially different from the continuous one. In this paper, we review the important advances so far in this direction, including the explosive synchronization in coupled oscillators with degree-frequency correlation, the explosive synchronization in frequency-weighted Kuramoto model, the explosive synchronization in coupled conformists and contrarians, the explosive synchronization in adaptive and multilayer networks, as well as the microscopic mechanism of the explosive synchronization and the experimental verification. These work not only have greatly enhanced our understandings of synchronization in complex systems and promoted the development of synchronization theory, but also paved a way for potential applications in future.
\end{abstract}

Kuramoto model, synchronization, explosive synchronization, phase transition, complex network

PACS: 05.45.Xt, 68.18.Jk, 89.75.-k

doi: 10.1360/SSPMA-2019-0135 